\title{
Outcome of single burr hole under local anesthesia in the management of chronic subdural hematoma
}

\author{
Hosni Salama
}

\begin{abstract}
Background: Management of chronic subdural hematoma (CSDH) is usually surgical; burr hole craniostomy using double burr hole under general anesthesia is the most commonly used technique for its evacuation. However, single burr hole under local anesthesia may be an alternative deserving the study. The aim of this work is to evaluate the prognosis of single burr hole under local anesthesia in the management of CSDH according to the etiology, clinical picture, surgical technique, and prognosis.
\end{abstract}

Patients and methods: We studied 67 consecutive patients with CSDH operated by single burr hole craniostomy under local anesthesia from March, 2014, to February, 2015, at Zagazig University Hospitals according to the etiology, clinical picture, surgical technique, and complications. Exclusion criteria were age below 19 years, multilocular hematomas, and uncooperative patients. This is a prospective clinical study.

Results: The age range was from 19 to 90 years with mean age of 60 years. There were 38 (56.7\%) males and 29 (43.3\%) females. The main cause of CSDH was old trauma in 43 (64.2\%), liver disease in 14 (20.9\%), antiplatelet in 8 (11.9\%), and ICP hypotension in 2 (3\%). Headache was found in 28 (41.8\%), disturbed conscious level in 19 (25.4\%), hemiparesis in 42 (62.7\%), and seizure in 6 (9\%). Markwalder scoring for CSDH at time of admission was $3(4.48 \%)$ in grade 0, 20 (29.85\%) in grade1, 23 (34.33\%) in grade 2, 17 (25.37\%) in grade 3, and 4 (5.97\%) in grade 4. After evacuation, Markwalder score was $53(79.01 \%)$ in grade 0, $13(19.4 \%)$ in grade 1, $1(1.50 \%)$ in grade 2, 0\% in grade 3, and $0 \%$ in grade 4.

Conclusion: Management of chronic subdural hematoma by single burr hole using local anesthesia is safe, effective, and sufficient for patient with unilocular cooperative CSDH patients with short hospital stay, low cost, and lower complication rate.

Trial registration: ClinicalTrials.gov Identifier: NCT03447327 registered on 26 February 2018. Retrospectively registered.

Keywords: Burr hole, Chronic subdural hematoma, Local anesthesia, Prognosis

\section{Introduction}

Chronic subdural hematoma (CSDH) is an encapsulated collection of old blood or blood breakdown products, partially or completely liquefied and located between the dura and arachnoid mater present at least for 3 weeks [1]. It represents 1.5 to 3 cases per 100,000 population per year [2].
Correspondence: hosni10565@yahoo.com

Faculty of Medicine, Zagazig University, Orabi Street, Zagazig, Sharkia Governorate, Egypt
Presentation of CSDH varies from patient to patient [3]. Atrophic brain associated with large subdural space predisposes the elderly people to the development of CSDH [4]. Advanced age also has the following predisposing factors: increased motor deficits, abnormal gait, increased capillary fragility, and cerebral atrophy [5]. Coexisting systemic disease usually poses a problem for general anesthesia in this particular patient group [6].

The clinical symptoms of CSDH may vary from no symptoms to unconsciousness; it may be diagnosed by computerized tomography $(\mathrm{CT})$ brain but magnetic 
resonance imaging (MRI) is more sensitive in isodense bilateral hematomas [2].

Computerized tomography (CT) scanning is usually sufficient for the diagnosis where it appears as crescent-shaped hypo-dense with concave surface towards the brain. Preoperative magnetic resonance imaging (MRI) is not carried out in most patients with CSDH but can help in doubtful cases [7].

Treatment of subdural hemorrhage could be conservative if there is insignificant amount of blood that is not compromising the cerebral cortex with no clinically significant manifestations. In spite of this, management of $\mathrm{CSDH}$, however, is usually surgical and frequently done by one of three procedures; twist drill craniostomy (opening less than $0.5 \mathrm{~cm}$ ), burr hole craniostomy $(0.5-$ $3 \mathrm{~cm}$ ), and craniotomy [8]. Of the three procedures, burr hole craniostomy is the most commonly used technique for evacuation of subdural hemorrhage. This is because of the high rate of recurrence in twist drill procedure and higher morbidity and mortality of the craniotomy technique [9].Selection bias may contribute to the increased morbidity and mortality of craniotomy technique that is because those patients selected for craniotomy were those patients with high risks for recurrence and mortality [10-13].

The aim of this work is to evaluate the prognosis of single burr hole under local anesthesia in the management of CSDH according to clinical picture, surgical technique, and prognosis.

\section{Methods}

We prospectively studied 67 consecutive patients with CSDH operated by single burr hole craniostomy under local anesthesia from March, 2014, to February, 2015, at Zagazig University Hospitals according to the etiology, clinical picture, medical disease and risk factors, and complications.

The information concerning etiologies, symptoms and signs, concomitant diseases, diagnosis, therapies, and outcomes were collected retrospectively by reviewing the clinical records.

Exclusion criteria included age below 19 years, multilocular hematomas, and uncooperative patients.

All the diagnosis was determined based on computed tomography (CT); CSDH was classified into hypodense hematomas, varying density hematomas and isodense or slightly hyperdense hematomas.

Informed consents were obtained from patients or their relatives. Burr hole craniostomy was performed under local anesthesia in all patients. Local anesthesia was used in all cases with $10 \mathrm{ml}$ Lidocaine $\mathrm{Hcl} 2 \%$ injected subperiosteally and subcutaneously; sedation with midazolam or propofol was used only in some irritable patients. Scalp incision is $3 \mathrm{~cm}$ down to the periosteum then periosteal cutting by diathermy then exposure of the skull. Single burr hole with $1.5 \mathrm{~cm}$ diameter was done, followed by dural cauterization, and then durotomy using no.11 knife and circular dural flap opposite the whole burr hole. Drainage of the subdural space with irrigation is done with warm saline until clear fluid comes, followed by the insertion of no.12 Nelaton catheter drain connected to a closed bag not under tension for $48 \mathrm{~h}$. Closure of the wound is after the hemostasis of the scalp edges. The patient was transferred directly to the ward in a flat position with plenty of IV fluids, and oral feeding is allowed directly. Follow-up of the patients clinically and radiologically was used for 6 months.

Patients' neurological status on admission and at discharge was classified on the basis of a grading scheme for CSDH suggested by Markwalder et al. [14]: grade 0, no neurological deficits; grade 1 , alert and oriented, mild symptoms such as headache, absent, or mild neurological deficits such as reflex asymmetry; grade 2, drowsy or disoriented with variable neurological deficits such as hemiparesis; grade 3, stuporous but responding appropriately to noxious stimuli, severe focal signs such as hemiplegia; and grade 4, comatose with absent motor response to painful stimuli and decerebrate or decorticate posturing (Fig. 1.

Glasgow outcome scale was used in conjunction with Markwalder grading but we preferred the latter due to more specificity in CSDH. Patients with no or mild symptoms are considered to have good outcome [15]while those who were unchanged or worsened are considered to have poor outcome [16].

\section{Results}

The age range was from 19 to 90 years with mean age of 60 years. There were 38 (56.7\%) males and 29 (43.3\%) females (Table 1).

The main cause of CSDH was old trauma in 43 (64.2\%) followed by liver disease in 14 (20.9\%), antiplatelet in 8 (11.9\%), and in ICP hypotension after lumbar puncture or V-P shunt insertion in $2(3 \%)$.

Clinically, headache was found in 28 (41.8\%), disturbed conscious level in 19 (25.4\%), hemiparesis in 42 (62.7\%), and seizure in $6(9 \%)$.

CT was low density in 41 (61.2\%), isodense in 5 (7.4\%), high density in $7(10.4 \%)$, and mixed in 14 (20.8\%). There were 27 (40.3\%) of patients who had the right side, $29(43.3 \%)$ in the left side, and $11(16.4 \%)$ in bilateral CSDH (Table 2).

Radiologic follow-up showed resolution of the CSDH in all cases with minimum residual in the early postoperative period and complete resolution at late follow-up except two recurrent cases at 4 and 6 weeks respectively and one case of empyema, all were managed by 


\section{Grade 0 arade 1 arade 2 arade $3 \square$ Grade 4}

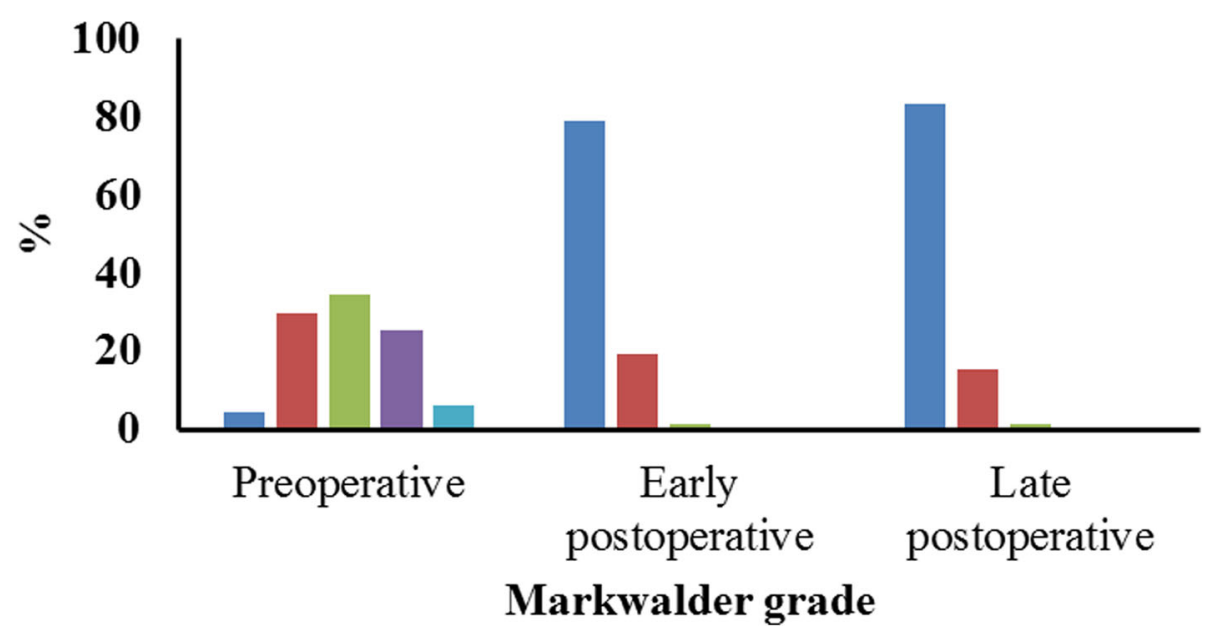

Fig. 1 Distribution of the patients according to Markwalder grade in preoperative, early, and late postoperative evaluations. The figure shows the relation between the Markwalder scale and the number of cases in each category. We can observe the marked improvement in Markwalder scale in both early and late postoperative follow-up

re-exploration of the same burr hole with good results. One case of moderate pneumocephalus was managed conservatively.

All patients were examined neurologically using Markwalder scoring for $\mathrm{CSDH}$ at the time of admission which was $3(4.48 \%)$ in grade $0,20(29.85 \%)$ in grade 1,23 $(34.33 \%)$ in grade $2,17(25.37 \%)$ in grade 3 , and 4 (5.97\%) in grade 4.The mean Markwalder grade was 2.

After evacuation, the Markwalder score dramatically improved where it was found in 53 (79.01\%)in grade 0 , $13(19.4 \%)$ in grade $1,1(1.50 \%)$ in grade $2,0 \%$ in grade 3 , and $0 \%$ in grade 4 . The mean Markwalder grade was 0 (Table 3).

After the surgical evacuation, two cases $(2.98 \%)$ had recurrent $\mathrm{CHDH}$, one case $(1.5 \%)$ had tension pneumocephalus, one case (1.5\%) suffer from empyema, and one case $(1.5 \%)$ died because of chest infection 2 weeks postoperatively. The outcome was good in $98.5 \%$ of patients; no poor outcome and death in $1.5 \%$ after 2 weeks.

\section{Discussion}

Chronic subdural hematoma is a common neurosurgical problem in old age, because most of these patients are fragile geriatric group; the least invasive technique is preferred. Burr hole craniostomy is considered the gold

Table 1 Demographic data of the patients

\begin{tabular}{llc}
\hline Demographic data & No. & Percent \\
\hline Age & $19-90$ & \\
Sex: Male & 38 & 56.7 \\
$\quad$ Female & 29 & 43.3 \\
\hline
\end{tabular}

standard for surgical treatment of chronic subdural hematoma $[17,9,14]$.

Our aim is to minimize the surgical manipulations and anesthetic agents and to enhance recovery with minimal complications and reduce recurrence rate.

In general, for patients who have coexisting complex systemic disease, local anesthesia is a more favored method during surgery for CSDH $[18,19]$.

Many reports from different studies noted the safety of both general and local anesthesia in chronic subdural hematoma with minor complications [16, 19-21].

Moreover, general anesthesia may alter the return to preoperative levels of consciousness after such procedures which need to be evaluated early postoperatively to exclude the need for redo due to early postoperative recollection [6].

Local anesthesia was used during endoscopic removal of clotted blood and in multilocular hematomas in fragile patients [22]. It was used by many other authors and Khadka et al. [23], Lee et al. [21], and Yadav et al. [2].

In our study, the mean age was 60 years old, and this is explained in most studies due to the known pathologic changes in this age group [2].

Our study showed male predominance $56.7 \%$, it was $71.8 \%$ males in the series of Mori and Maeda [16] and 66.3\% in Ernestus et al. [15]. The predominance of male sex was due to the more vulnerability for trauma.

Regarding the location of CSDH, the left side was the most commonly affected in our study and in most other studies, 52\% in Mori and Maeda [16]. The explanation may be the more convex skull on the left side in most people [24]. 
Table 2 Clinical and radiologic presentation

\begin{tabular}{llll}
\hline Etiology & Trauma & 43 & 64.2 \\
& Antiplatelet & 8 & 11.9 \\
& Liver disease (coagulopathy) & 14 & 20.9 \\
Clinical & ICP hypotension & 2 & 3.0 \\
& Headache & 28 & 41.8 \\
& Hemiparesis & 42 & 62.7 \\
& DC (disturbed consciousness) & 17 & 25.4 \\
CT & Seizure & 1 & 1.5 \\
& Low & 41 & 61.2 \\
& Iso & 5 & 7.4 \\
& High & 7 & 10.4 \\
Sidedness & Mixed & 14 & 20.8 \\
& Right & 27 & 40.3 \\
& Left & 29 & 43.3 \\
& Bilateral & 11 & 16.4 \\
\hline
\end{tabular}

Clinical features develop over a period of days to weeks. Sometimes, patients fail to recall events of head injury. The common predisposing factors are head injury, alcoholism, seizure disorders, brain atrophy, anticoagulation, and impaired surgical hemostasis. Head injury was evident in $64.2 \%$ of our patients. It is usually a trivial trauma [2].

Clinically, patient may present with headache, nausea, hemiparesis, vomiting, sensory deficit, language disturbance, gait problems, transient ischemic symptoms, convulsions, decreased level of consciousness, and raised intra cranial pressure. In our study, the clinical presentation included hemiparesis, disturbed conscious level, headache, and rarely seizures.

The most common symptom was hemiparesis followed by gait disturbance; headache was common in patients at younger ages, and this is explained by the tendency to have increased intracranial pressure at younger ages [16].

The surgical techniques used for subdural hematoma evacuation vary from twist drill craniostomy to large craniotomy procedures $[20,15]$. Our choice of single burr hole instead of double burr hole was based on the assumption that unilocular CSDH could be efficiently drained and washed without the need of extra burr hole as long as it is a single fluid containing cavity.
The mean operative time was $35 \mathrm{~min}$ in our study, this time is equal to that of Gozel et al. [6] (36.4 min with a range from 25 to $63 \mathrm{~min}$ ), and this is another advantage for our technique.

The number of recurrence in our study was very low, only two cases (2.98\%), and this is one of the best results reported in chronic subdural hematoma surgery; the rate of recurrence after burr hole evacuation and irrigation varies between 2.7 and 34\% [14, 16, 23, 25, 26].

The rate of recurrence was related to the amount of subarachnoid air in most series. Poor re-expansion of the brain is correlated to hematoma recurrence; therefore, air influx into the subdural space should be prevented during surgery [16]. Tension pneumocephalus was a dangerous complication to be prevented by filling the subdural space by normal saline, so we think that the absence of tension pneumocephalus and the lower rate of recurrence were due to the capability to fill the subdural space with saline with single burr hole than with two burr holes as there is no leak from the second burr hole during filling from the first one.

The rate of acute subdural hematoma formation was nil; this is due to the small single scalp incision and the ability to control scalp bleeding easily.

By following the patients for 6 months, the operative complications were very limited; two recurrent cases and one died after 2 weeks due to chest infection.

Two cases developed postoperative seizures, and one of them was having the convulsions preoperatively; the second one suffered the first postoperative attack 1 week postoperatively and was controlled within 1 week by anticonvulsants (IV phenytoin for 3 days followed by oral phenytoin for 2 years).

Epilepsy is traditionally thought to be a rare presentation, even though it has been reported in up to $6 \%$ of cases as an initial symptom [27].

\section{Conclusion}

Management of chronic subdural hematoma by single burr hole using local anesthesia is safe, effective, and sufficient for patient with unilocular cooperative CSDH patients with short hospital stay, low cost, and lower complication rate.

Table 3 Markwalder grading of chronic subdural hematoma patients before and after surgery

\begin{tabular}{lllllll}
\hline Markwalder grade & Preoperative no. & Percent & Early postoperative no. & Percent & Late postoperative no. & Percent \\
\hline Grade 0 & 3 & 4.48 & 53 & 79.10 & 55 & 83.3 \\
Grade 1 & 20 & 29.85 & 13 & 19.40 & 10 & 15.2 \\
Grade 2 & 23 & 34.33 & 1 & 1.50 & 1 & 1.5 \\
Grade 3 & 17 & 25.37 & 0 & 00 & 0 & 00 \\
Grade 4 & 4 & 5.97 & 0 & 00 & 0 & 00 \\
$\quad$ Total & 67 & 100 & 67 & 100 & 66 & 100 \\
\hline
\end{tabular}




\section{Abbreviations}

CSDH: Chronic subdural hematoma; CT: Computerized tomography; DC: Disturbed consciousness; MRI: Magnetic resonance imaging

\section{Acknowledgements}

Not applicable

\section{Funding}

The research has no external funding.

\section{Availability of data and materials}

Please contact author for data requests.

\section{Author's contributions}

The single author of this article was responsible for performing the surgery, data collection and analysis, and writing of the manuscript. The author read and approved the final manuscript

\section{Ethics approval and consent to participate}

This study was approved by the Zagazig University Institutional Review. Board Zu-IRB at 3 March 2014. Written consent from all patients was taken before surgery.

\section{Consent for publication}

Not applicable

\section{Competing interests}

The author declares that he has no competing interests.

\section{Publisher's Note}

Springer Nature remains neutral with regard to jurisdictional claims in published maps and institutional affiliations.

Received: 6 September 2018 Accepted: 9 January 2019

Published online: 31 January 2019

\section{References}

1. Agrawal A. Bilateral biconvex frontal chronic subdural hematoma mimicking extradural hematoma. Journal of surgical technique and case report. 2010; 2(2).

2. Yadav YR, Parihar V, Namdev H, Bajaj J. Chronic subdural hematoma. Asian journal of neurosurgery. 2016 Oct;11(4):330.

3. Delgado-Lopez PD, Martin-Velasco V, Castilla-Diez JM, Rodríguez-Salazar A, Galacho-Harriero AM, Fernández-Arconada O. Dexamethasone treatment in chronic subdural haematoma. Neurocirugia. 2009 Jan 1;20(4):346-59.

4. Yang Al, Balser DS, Mikheev A, Offen S, Huang JH, Babb J, Rusinek H, Samadani U. Cerebral atrophy is associated with development of chronic subdural haematoma. Brain Inj. 2012 Dec 1:26(13-14):1731-6.

5. Tagle P, Mery F, Torrealba G, Del Villar PM, Carmona H, Campos M, Méndez J, Chicharro A. Hematoma subdural crónico: una enfermedad del adulto mayor. Rev Med Chil. 2003 Feb;131(2):177-82.

6. Guzel A, Kaya S, Ozkan U, Aluclu MU, Ceviz A, Belen D. Surgical treatment of chronic subdural haematoma under monitored anaesthesia care. Swiss Med Wkly. 2008 Jul 12;138(27-28):398-403.

7. Lee KS, Shim JJ, Yoon SM, Doh JW, Yun IG, Bae HG. Acute-on-chronic subdural hematoma: not uncommon events. Journal of Korean Neurosurgical Society. 2011 Dec;50(6):512

8. Weigel R, Schmiedek P, Krauss JK. Outcome of contemporary surgery for chronic subdural haematoma: evidence based review. J Neurol Neurosurg Psychiatry. 2003 Jul 1;74(7):937-43.

9. Wakai S, Hashimoto K, Watanabe N, Inoh S, Ochiai C, Nagai M. Efficacy of closed-system drainage in treating chronic subdural hematoma: a prospective comparative study. Neurosurgery. 1990 May 1;26(5):771-3.

10. Piotrowski WP, Krombholz-Reindl MA. Surgical outcome in chronic subdural hematoma. Unfallchirurgie. 1996 Jun;22(3):110-6.

11. Probst C. Peritoneal drainage of chronic subdural hematomas in older patients. J Neurosurg. 1988 Jun;68(6):908-11.

12. Robinson RG. Chronic subdural hematoma: surgical management in 133 patients. J Neurosurg. 1984 Aug;61(2):263-8.

13. Tanikawa M, Mase M, Yamada K, Yamashita N, Matsumoto T, Banno T, Miyati T. Surgical treatment of chronic subdural hematoma based on intrahematomal membrane structure on MRI. Acta Neurochir. 2001 Jun 1; 143(6):613-9.

14. Markwalder TM, Steinsiepe KF, Rohner M, Reichenbach W, Markwalder H. The course of chronic subdural hematomas after burr-hole craniostomy and closed-system drainage. J Neurosurg. 1981 Sep;55(3):390-6.

15. Ernestus Rl, Beldzinski P, Lanfermann H, Klug N. Chronic subdural hematoma: surgical treatment and outcome in 104 patients. Surg Neurol. 1997 Sep 1;48(3):220-5.

16. MORI K, MAEDA M. Surgical treatment of chronic subdural hematoma in 500 consecutive cases: clinical characteristics, surgical outcome, complications, and recurrence rate. Neurol Med Chir 2001:41(8):371-381.

17. Drapkin AJ. Chronic subdural hematoma: pathophysiological basis for treatment. Br J Neurosurg. 1991 Jan 1:5(5):467-73.

18. Deng XM, Xiao WJ, Luo MP, Tang GZ, Xu KL. The use of midazolam and small-dose ketamine for sedation and analgesia during local anesthesia. Anesth Analg. 2001 Nov 1;93(5):1174-7.

19. Rohde V, Graf G, Hassler W. Complications of burr-hole craniostomy and closed-system drainage for chronic subdural hematomas: a retrospective analysis of 376 patients. Neurosurg Rev. 2002 Mar 1;25(1-2):89-94.

20. Erol FS, Topsakal C, Ozveren MF, Kaplan M, Tiftikci MT. Irrigation vs. closed drainage in the treatment of chronic subdural hematoma. J Clin Neurosci. 2005 Apr 1:12(3):261-3.

21. Lee $\mathrm{JY}$, Ebel H, Ernestus Rl, Klug N. Various surgical treatments of chronic subdural hematoma and outcome in 172 patients: is membranectomy necessary? Surg Neurol. 2004 Jun 1;61(6):523-7.

22. Rodziewicz GS, Chuang WC. Endoscopic removal of organized chronic subdural hematoma. Surg Neurol. 1995 Jun 1;43(6):569-73.

23. Khadka NK, Sharma GR, Roka YB, Kumar P, Bista P, Adhikari D, Devkota UP. Single burr hole drainage for chronic subdural haematoma. Nepal Med Coll J. 2008 Dec;10(4):254-7.

24. Kim BG, Lee KS, Shim J, Yoon SM, Doh JW, Bae HG. What determines the laterality of the chronic subdural hematoma? Journal of Korean Neurosurgical Society. 2010 Jun;47(6):424

25. Tabaddor K, Shulman K. Definitive treatment of chronic subdural hematoma by twist-drill craniostomy and closed-system drainage. J Neurosurg. 1977 Feb;46(2):220-6

26. Aung TH, Wong WK, Mo HP, Tsang CS. Management of chronic subdural haematoma: burr hole drainage, replacement with Hartmann's solution, and closed-system drainage. Hong kong medical journal. 1999 Dec;5(4):383-6.

27. Adhiyaman V, Asghar M, Ganeshram KN, Bhowmick BK. Chronic subdural haematoma in the elderly. Postgrad Med J. 2002 Feb 1;78(916):71-5.

\section{Submit your manuscript to a SpringerOpen ${ }^{\bullet}$ journal and benefit from:}

- Convenient online submission

- Rigorous peer review

- Open access: articles freely available online

High visibility within the field

Retaining the copyright to your article

Submit your next manuscript at $>$ springeropen.com 